\title{
What Neural Correlates Underlie Successful Encoding and Retrieval? A Functional Magnetic Resonance Imaging Study Using a Divided Attention Paradigm
}

\author{
Elizabeth A. Kensinger, ${ }^{1}$ Richard J. Clarke, ${ }^{1}$ and Suzanne Corkin ${ }^{1,2}$ \\ ${ }^{1}$ Department of Brain and Cognitive Sciences, Massachusetts Institute of Technology, Cambridge, Massachusetts 02139, and ${ }^{2}$ Athinoula A. Martinos Center \\ for Biomedical Imaging, Charlestown, Massachusetts 02129
}

\begin{abstract}
If attention is divided during learning, memory suffers. Nevertheless, individuals can learn information with divided attention. This event-related functional magnetic resonance imaging (fMRI) study $(n=17)$ investigated what neural processes support (1) learning with divided attention and (2) retrieval of information learned with divided attention. Participants encoded words (Is the word abstract or concrete?) while performing an auditory discrimination task (press a button whenever an auditory pattern changes). The auditory task was easy or hard, depending on the similarity of the patterns. A behavioral study indicated that detailed ("recollective") information was more likely to be present for words encoded with the easy versus the hard concurrent task. Words encoded with the hard versus the easy concurrent task, in contrast, were more likely to rely on less detailed ("familiarity"-based) information. fMRI revealed encoding-related activation in the left prefrontal cortex (PFC) and left hippocampus that was linked to successful memory formation only for items encoded with the easy task. In contrast, activation in the right PFC and left parahippocampal gyrus was linked to successful memory for all items. Thus, successful encoding with the hard concurrent task was supported by a subset of the regions recruited for successful encoding with the easy task. The neural processes recruited for successful retrieval also depended on the encoding condition: The left PFC was disproportionately recruited for retrieval of items encoded with the easy task, whereas the right PFC was disproportionately recruited for retrieval of items encoded with the hard task. These findings may reflect left-sided specialization for recollective memories and right-sided specialization for familiarity-based traces.
\end{abstract}

Key words: divided attention; explicit memory; encoding; retrieval; dual task; neuroimaging; prefrontal cortex; human

\section{Introduction}

The term "divided attention" refers to an experimental paradigm in which participants learn information while performing a concurrent task that is easy (taking few resources from the encoding operation) or hard (shifting resources from the encoding operation). [We remain neutral as to whether the divided attention manipulation at encoding taxes a general attention system or a working memory system (or these two systems may be equivalent).] Participants' memory is worse for items encoded with a hard than with an easy secondary task (Baddeley, 1984; Craik et al., 1996; Naveh-Benjamin et al., 2000a,b). Positron emission tomography (PET) studies using a divided-attention paradigm found less activation in regions of prefrontal cortex (PFC) with performance of a hard secondary task versus an easy one (Shallice et al., 1994; Fletcher et al., 1995, 1998; Anderson et al., 2000; Iidaka et al., 2000). The PFC regions in which activation was affected are those that have been linked to episodic encoding (Fletcher et al., 1998; Wagner et al., 1998; Kirchhoff et al., 2000; Paller and Wagner, 2002). These regions may have shown greater activation during encoding with the easy versus the hard task

\footnotetext{
Received Sept. 4, 2002; revised Dec. 3, 2002; accepted Dec. 27, 2002.

This work was supported by National Institutes of Health Grant AG14432, the National Science Foundation, and a Howard Hughes Medical Institute Predoctoral Fellowship (E.A.K.)

Correspondence should be addressed to Elizabeth A. Kensinger, NE20-392, 77 Massachusetts Avenue, Cambridge, MA 02139. E-mail: ekensing@mit.edu.

Copyright $\odot 2003$ Society for Neuroscience $\quad 0270-6474 / 03 / 232407-09 \$ 15.00 / 0$
}

because some encoding-related processes were not performed with concurrent performance of a hard secondary task (Shallice et al., 1994; Fletcher et al., 1995; Iidaka et al., 2000).

Despite evidence that encoding processes are disrupted by divided attention, individuals can successfully encode some information while performing a hard secondary task. This finding raises the following question: What neural substrates support successful memory formation when resources for encoding are reduced (i.e., during concurrent performance of a hard task)? [The requisite blocked design of PET prevented investigation of this issue. The event-related functional magnetic resonance imaging (fMRI) design of this study allows correlation of single events during encoding with later retrieval, thus providing a way to address this question.] One possibility is that the same regions that are usually implicated in successful memory formation (Brewer et al., 1998; Fernandez et al., 1999; Wagner et al., 1999; Kirchhoff et al., 2000; Fernandez and Tendolkar, 2001; Otten and Rugg, 2001; Paller and Wagner, 2002) continue to support successful encoding during performance of a hard concurrent task. An alternate option is that only a subset of the regions that typically support successful encoding continue to do so during performance of a hard task.

To understand why this second option could occur, consider that individuals can form two kinds of memories: (1) vivid, detailed memories that they can "remember" or "recollect," or (2) memories that lack detail and provide only a feeling that the item was presented (sense of "knowing" or "familiarity") (Mandler, 
1980; Tulving, 1985; Jacoby, 1991; Gardiner and Java, 1993; Yonelinas, 2002). Formation of vivid, "recollective" memories is thought to require more attention than formation of less detailed traces; thus, divided attention may disproportionately affect the encoding of detailed memories (Yonelinas, 2001, 2002). [Shallice et al. (1994) and Fletcher et al. (1995) have proposed that divided attention may disrupt the ability to form explicit memories, while allowing formation of implicit memories.] Activation in the subset of brain regions that allows less detailed memory formation may relate to successful encoding even with divided attention, whereas activation in regions that support detailed memory formation may not.

If divided attention qualitatively alters the memories formed, then the divided attention manipulation could affect not only the neural processes recruited for successful memory formation but also those recruited for successful retrieval. Retrieval of detailed, recollective memories may rely more on the left PFC (Johnson et al., 1997; Nolde et al., 1998a,b; Henson et al., 1999a,b; Rugg et al., 1999) and the hippocampus (Eldridge et al., 2000; Davachi et al., 2001), while retrieval of familiarity-based memories may rely on the right PFC (Henson et al., 1999a,b; Eldridge et al., 2000) and the parahippocampal gyrus (Strange et al., 2002; Davachi et al., 2003). Therefore, we hypothesized that the left PFC and left hippocampus would be disproportionately recruited for retrieval of words encoded with the easy concurrent task, whereas the right PFC and left parahippocampal gyrus would be disproportionately recruited during retrieval of words encoded with the hard concurrent task.

\section{Materials and Methods}

Behavioral companion study

To confirm that the difficulty of the secondary task at encoding affected the amount of detail that could be retrieved, we conducted a behavioral study outside of the scanning environment.

Participants. Participants in this experiment comprised 24 young adults (12 women). They were matched to participants in the imaging study by age (20 - 35 years of age; mean, 26.4) and education level (14-18 years; mean, 16.3).

Encoding. The stimuli were 480 words, with written frequencies ranging from 10 to 100 (Kucera and Francis, 1967). They were presented one at a time in Geneva 48 point font, using MacStim (David Darby, WhiteAnt Occasional Publishing, West Melbourne, Victoria, Australia). Stimulus words were divided into four encoding runs, each with 120 words.

Participants completed two encoding tasks, each followed by a retrieval task. In the encoding task, participants saw words for 2 sec each and rated each word as abstract or concrete by pressing a button with their right middle or ring finger, respectively. Because we wanted the behavioral encoding task to be identical to that performed in the scanner, fixation crosses were interspersed pseudorandomly between the words. Participants made no response when fixation occurred.

In addition to making the abstract-concrete decisions, participants simultaneously performed an auditory discrimination task. For this task, $1.5 \mathrm{sec}$ auditory patterns (created using Sound Edit; MacroMedia, Inc., San Francisco, CA) were presented continuously as people rated words and also when they viewed the fixation crosses. The participants' task was to listen to these patterns and to press a button with their left index finger every time a sound pattern changed from pattern A to pattern B (or from $\mathrm{B}$ to A). The difficulty of the auditory discrimination task was related to the similarity of auditory patterns A and B. In the easy version of the task, the two auditory patterns were rhythmically distinct and thus easy to discriminate. In the hard version, the patterns were rhythmically similar and hard to discriminate. Participants were given an instructional cue as to whether the secondary task would be easy or hard, and that task version continued for $30 \mathrm{sec}$, until the next instructional cue (Fig. 1).

Retrieval. After each of the encoding tasks, participants performed a recognition task with 72 previously presented words and 48 nonpre-

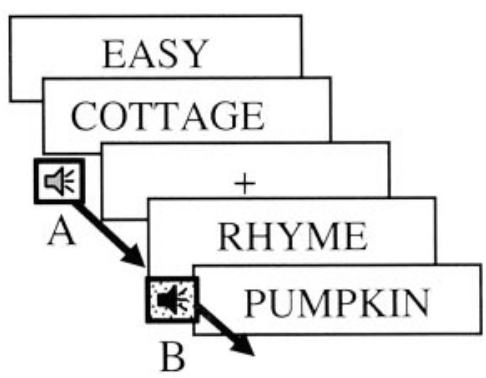

Figure 1. The instruction frame and first 8 sec of a representative encoding pseudoblock. $A 2$ sec instruction frame indicated the encoding task (classify words as abstract or concrete) and difficulty of the auditory discrimination task (easy or hard). Words were presented for 2 sec each and were pseudorandomly intermixed with fixation crosses. Sound patterns played throughout the pseudoblock, and participants pressed a button when they noted that the sound pattern changed (e.g., from pattern A to pattern B).

sented words. Pilot data suggested that with this breakdown, participants responded that approximately one-half of the stimuli were presented previously. Participants were asked to indicate via button press whether they (1) "remembered" the word from the encoding list, (2) "knew" the word was presented previously, or (3) thought the word was "new" (nonpresented). A "remember" response indicated that participants had a vivid memory of the presented item, whereas a "know" response indicated that participants lacked a specific memory of the presentation of the item but sensed that it had been presented recently (Tulving, 1985; Jacoby, 1991).

Data analysis. Data were analyzed in two ways. First, we calculated the corrected recognition scores (percentage of remember hits - percentage of remember false alarms or percentage of know hits - percentage of know false alarms). Second, we computed recollection and familiarity scores as suggested by Yonelinas et al. (1998). These scores take into account the fact that the probability of making a know response to a presented word is constrained by the number of remember responses made to presented words, because participants were instructed to give a know response to items that are familiar and not recollected. Analyses consisted of repeated-measures ANOVA with secondary task type (easy, hard) and memory strength (remember, know; recollection, familiarity) as within-subject factors, and subsequent $t$ tests. All reported $p$ values are two-tailed.

\section{fMRI study}

Having confirmed in the behavioral study that the manipulation of secondary task difficulty affected overall memory as well as the richness of the memory traces formed, we then examined what effect this manipulation of secondary task difficulty would have on the neural processes underlying successful encoding and retrieval.

Participants. The participants comprised 22 right-handed, native English speakers. Five participants were excluded because of scanner malfunction (one man, one woman), sound presentation failure (one man), or excessive head movement (one man, one woman). The remaining 17 participants (nine men, eight women) were 20-35 years of age (mean age, 26.1) with $14-18$ years of education (mean, 16.0 years). Participants were screened to exclude those with any history of neurological or psychiatric disorder, and no participants were taking centrally acting medications.

General methods. Before entering the scanner, participants performed a practice encoding and retrieval run. Once in the scanner, they completed an additional short practice run to allow familiarization with the presentation of the sound patterns via headphones. Participants were scanned during three encoding and three retrieval runs.

Encoding. The encoding task was identical to that described in the behavioral study. Each encoding run included 1230 sec pseudoblocks; each pseudoblock consisted of 10 words, each presented for $2 \mathrm{sec}$. The times of the word onsets were jittered with fixation crosses to allow for optimal accuracy and efficiency of estimation of the hemodynamic response to each stimulus (Dale et al., 1999). We used the optseq program 
(part of the FS-FAST analysis tools; http://surfer.nmr.mgh.harvard.edu/ optseq, written by D. Greve, Charlestown, MA), which determines stimulus optimization (i.e., the optimal word onsets) given the repetition time (TR), number of event types, time per event type, and number of acquisitions. A description of the calculations used in the development and implementation of this stimulus optimization program can be found in previous publications (Burock et al., 1998; Dale, 1999).

Although task type was blocked (i.e., participants performed either the hard distractor task or the easy distractor task for $30 \mathrm{sec}$ periods), we could still resolve the hemodynamic response associated with each individual item because the items were jittered with fixation (Burock et al., 1998; Dale, 1999). Therefore, the analysis of the data was event-related.

Retrieval. A recognition run followed each encoding run. Each recognition run included 120 words, with 60 fixation points pseudorandomly intermixed to provide jitter. Across the recognition runs, 108 words had been encoded in the easy discrimination condition (one-half retrieved in the hard condition; one-half retrieved in the easy condition); 108 had been encoded in the hard discrimination condition (one-half retrieved in the hard condition; one-half retrieved in the easy condition); and 144 new words were pseudorandomly intermixed (one-half in the easy condition). The order of the encoding-recognition runs was counterbalanced across the participants.

Control experiment. In a control experiment, nine young adults (six males, 18-30 years of age) performed only the auditory discrimination task (without the encoding or retrieval tasks). As in the main experiment, an instructional cue informed the participants whether the task would be easy or hard, and the tasks were presented in $30 \mathrm{sec}$ blocks. In addition, there was a "no task" condition, which served as the baseline. Throughout all task conditions, participants viewed a fixation cross on the screen in addition to monitoring the sound patterns. The critical comparison was between the hard task and the easy task conditions.

Scanning protocols. Imaging was performed on a Siemens (Erlangen, Germany) Allegra 3 Tesla head-only MRI scanner with a $36 \mathrm{~cm}$ inner diameter asymmetric gradient coil. Head motion was minimized using pillows and foam padding around the head. For each participant, 2 magnetization-prepared rapid acquisition gradient echo structural scans optimized for gray/white contrast were collected to provide detailed anatomical information. After the structural scans, a series of echoplanar functional scans was collected to provide images sensitive to blood oxygen level-dependent (BOLD) contrast. Functional T2*-weighted images were acquired using a single-shot echoplanar sequence (tau, $-25 \mathrm{msec}$; echo time, $30 \mathrm{msec}$; TR, $2000 \mathrm{msec} ; 3.13 \mathrm{~mm}$ in-plane resolution; $5 \mathrm{~mm}$ slice thickness; no skip). Slices were aligned along the anterior commisure/ posterior commisure line. Each scan lasted $6 \mathrm{~min}$ and $24 \mathrm{sec}$, during which 92 images were acquired in an interleaved manner for each of 22 axial slices.

Data analysis. Data were motion corrected using the Analysis of Functional Neuroimages motion correction algorithm (Cox and Jesmanowicz, 1999). We excluded participants whose motion exceeded two functional voxels. We conducted analyses both with and without global signal intensity normalization. The results did not differ qualitatively; we therefore report the normalized data here.

We used selective averaging and deconvolution of the BOLD signal. The BOLD signal was modeled as a linear combination of hemodynamic responses, offset, and trend embedded in Gaussian nose (Boynton et al., 1996; Dale and Buckner, 1997; Dale, 1999). The hemodynamic response to a particular stimulus type was modeled as a sum of $\Delta$ functions over a fixed time window phase-locked to the presentation of the stimulus. No response shape for this function was assumed, but it was assumed that the response to a particular stimulus type was the same for every presentation of that stimulus type, and that the overlap of temporally adjacent responses was linear. This signal model was inverted to obtain the average hemodynamic response to each stimulus as if that stimulus were presented in isolation, allowing for the resolution of individual events (Boynton et al., 1996; Dale et al., 1997; Dale, 1999).

Effects for each condition were estimated using a participant-specific, fixed-effects model. The participant-specific effects from each of these contrasts were entered into a second-level group analysis treating participants as a random effect. Regions were considered reliable if they con-
Table 1. Corrected recognition performance as a function of encoding condition in the behavioral companion study (mean, SD)

\begin{tabular}{lll}
\hline & Remember & Know \\
\hline Encoding with easy distractor & $43(18)$ & $14(28)$ \\
Encoding with hard distractor & $28(12)$ & $24(21)$ \\
\hline
\end{tabular}

sisted of at least five contiguous voxels that exceeded a threshold of $p<$ 0.001 (uncorrected).

Regions of interest (ROI) were defined functionally based on the contrasts of all encoding versus baseline or all retrieval versus baseline. The defined ROIs were therefore unbiased with respect to the contrast of interest (easy vs hard concurrent task; remembered vs forgotten). ROI analyses examined whether there was a reliable condition (e.g., words remembered, words forgotten) by time $(0-14 \mathrm{sec})$ interaction. Time represented the time course of the signal change; thus, an interaction between condition and time indicated that the signal change differed based on condition during a specific part of the time course (i.e., the interaction indicated that the condition effect was not related to a nonspecific elevated signal change). Planned contrasts also examined whether the peak percentage signal change (across $2 \mathrm{sec}$ time points, compared with the fixation-sound baseline) differed for two conditions (e.g., remembered, forgotten or easy, hard).

We resampled the functional data into standardized space (Spherical, Talairach) to allow averaging of group data. Automatic spherical resampling (Dale et al., 1999; Fischl et al., 1999, 2001) examined the effect of condition on cortical activation for the group. Automatic Talairach resampling using the Montreal Neurological Institute atlas (Mazziotta et al., 1995) examined the effect of condition (e.g., hard versus easy encoding) on subcortical activation for the group of participants. All group data were analyzed using random-effects analyses (Friston et al., 1999).

\section{Results}

\section{Behavioral companion study}

Table 1 shows remember scores and know scores for each of the conditions. All scores were corrected for false alarm rate (percentage of hits - percentage of false alarms). Repeated-measures ANOVA, calculated either on the raw remember and know scores or on the computed recollection and familiarity scores (Yonelinas et al., 1998), indicated a significant effect of encoding diffi$\operatorname{culty}\left(F_{(1,23)}=6.4 ; p<0.01\right)$ and an interaction between memory strength (remember vs know or recollection vs familiarity) and encoding difficulty $\left(F_{(1,23)}=6.4 ; p<0.01\right)$.

The results of this behavioral companion study confirmed that the hard auditory task at encoding altered the type of memory trace formed. Items encoded with the hard auditory task were recognized by only a sense of previous encounter (know response) more frequently than items encoded with the easy auditory task. Items encoded with the easy auditory task, in contrast, were recognized based on detailed information about the presentation of the item (remember response) more frequently than were items encoded with the hard auditory task.

\section{fMRI study}

\section{Behavioral performance}

Accuracy. The presence of the hard secondary task at encoding led to poorer memory than did the presence of the easy secondary task $(t=5.1 ; p<0.01)$. In contrast, the difficulty of the secondary task at retrieval did not affect memory performance $(p>0.1)$. Repeated-measures ANOVA indicated no effect of memory process (encoding, retrieval) $\left(F_{(1,16)}=0.10 ; p>0.3\right)$, a significant effect of task difficulty $\left(F_{(1,16)}=4.42 ; p<0.01\right)$ on memory performance, and a significant task difficulty by memory process interaction $\left(F_{(1,16)}=5.25 ; p<0.01\right)$, indicating that the difficulty manipulation had a greater effect at encoding than at retrieval 
Table 2. Mean corrected (percentage of hits - percentage of false alarms) recognition performance (SD) as a function of secondary task difficulty and memory process

\begin{tabular}{lll}
\hline & \multicolumn{2}{c}{ Retrieval } \\
\cline { 2 - 3 } Encoding & Easy distractor & Hard distractor \\
\hline Easy distractor & $64(21)$ & $62(21)$ \\
Hard distractor & $56(16)$ & $57(22)$ \\
\hline
\end{tabular}

Table 3. Reaction times (in milliseconds) to the change in sound patterns as a function of secondary task difficulty and memory process (mean, SD)

\begin{tabular}{llll}
\hline $\begin{array}{l}\text { Encoding with easy } \\
\text { distractor }\end{array}$ & $\begin{array}{l}\text { Encoding with hard } \\
\text { distractor }\end{array}$ & $\begin{array}{l}\text { Retrieval with } \\
\text { easy distractor }\end{array}$ & $\begin{array}{l}\text { Retrieval with } \\
\text { hard distractor }\end{array}$ \\
\hline $510(220)$ & $930(210)$ & $600(170)$ & $980(180)$
\end{tabular}

Table 4. Reaction times (in milliseconds) to retrieve words as a function of secondary task difficulty at encoding and retrieval (mean, SD)

\begin{tabular}{lll}
\hline & \multicolumn{2}{c}{ Retrieval } \\
\cline { 2 - 3 } Encoding & Easy distractor & Hard distractor \\
\hline Easy distractor & $830(230)$ & $890(220)$ \\
Hard distractor & $850(200)$ & $870(290)$ \\
\hline
\end{tabular}

(Table 2). These results are consistent with the findings of numerous behavioral studies (Craik et al., 1996, 2000; Naveh-Benjamin et al., 1998; Naveh-Benjamin and Guez, 2000) and are consistent with the performance we found on this task in a behavioral pilot study.

Reaction times for noting sound pattern changes. A comparison of reaction times for the two auditory discrimination conditions indicated that participants were slower to respond to auditory pattern changes when the discrimination was hard than when it was easy (Table 3$)\left(F_{(1,16)}=733.32 ; p<0.0001\right)$. Participants were slower at indicating the sound pattern change (regardless of auditory task difficulty) when it occurred at retrieval than at encoding $\left(F_{(1,16)}=6.64 ; p<0.01\right)$, but there was no interaction between task difficulty and memory process $\left(F_{(1,16)}=0.91 ; p>\right.$ 0.2 ), indicating that the increased time to respond to the hard versus the easy auditory pattern changes was similar at encoding and retrieval. These results are also consistent with those of previous behavioral studies, as well as the performance of participants on this task when assessed outside of the scanner.

Reaction times for rating words as abstract/concrete or old/new. Participants' reaction times for rating the words as abstract or concrete were not affected by the difficulty of the auditory discrimination task at encoding, nor were the reaction times for making old or new decisions at retrieval affected by secondary task difficulty (Table 4). Repeated-measures ANOVA revealed no effect of task difficulty at encoding or at retrieval and no interaction between encoding difficulty and retrieval difficulty.

\section{fMRI results: encoding}

We first conducted voxel-based statistical analyses, which revealed the voxels of the brain that showed condition-related activity above a threshold of $p<0.001$. These analyses revealed that encoding with either the easy or the hard distractor task (compared with baseline) elicited activation in occipital, parietal, and frontal regions bilaterally, and in the left medial temporal lobe (MTL). Interestingly, the regions recruited were similar regardless of the difficulty of the secondary task: In a direct contrast of encoding with the hard distractor task versus encoding with the
Table 5. Brain regions showing significantly more activation during encoding with the easy task than during baseline

\begin{tabular}{llll}
\hline Brain region & Hemisphere & $\begin{array}{l}\text { Talairach coordinates } \\
(x, y, z)\end{array}$ & Brodmann area \\
\hline Superior frontal gyrus & Left & $-4,6,55$ & 6 \\
Middle frontal gyrus & Left & $-55,36,16$ & $45 / 46$ \\
Middle frontal gyrus & Left & $-43,28,28$ & $9 / 46$ \\
Inferior frontal gyrus & Left & $-32,24,10$ & 45 \\
Inferior frontal gyrus & Left & $-48,38,-12$ & 46 \\
Precentral gyrus & Left & $-28,-25,38$ & 4 \\
Postcentral gyrus & Left & $-48,-17,49$ & 1 \\
Superior parietal lobule & Left & $-28,-60,51$ & 7 \\
Inferior parietal lobule & Left & $-51,-22,19$ & 40 \\
Inferior temporal gyrus & Right & $40,-70,-10$ & 37 \\
Occipital gyrus & Left & $-20,-93,-2$ & 18
\end{tabular}

Thalamus Left

Regions were defined as significant if they included at least five functional voxels at an uncorrected significance of $p<0.001$.

Table 6. Brain regions showing significantly more activation during encoding with the hard task than during baseline

\begin{tabular}{lllc}
\hline Brain region & Hemisphere & $\begin{array}{l}\text { Talairach coordinates } \\
(x, y, z)\end{array}$ & Brodmann area \\
\hline Superior frontal gyrus & Left & $-4,3,59$ & 6 \\
Middle frontal gyrus & Left & $-51,36,16$ & 46 \\
Cingulate gyrus & Right & $4,36,20$ & 32 \\
Precentral gyrus & Left & $-47,-17,49$ & 4 \\
Postcentral gyrus & Left & $-60,-18,20$ & 1 \\
Superior parietal lobule & Right & $43,18,54$ & 7 \\
Inferior parietal lobule & Left & $-35,-36,46$ & 7 \\
Inferior temporal gyrus & Left & $-35,-47,-18$ & 37 \\
Occipital gyrus & Left & $-8,-93,1$ & 18 \\
Thalamus & Right & &
\end{tabular}

Regions were defined as significant if they included at least five functional voxels at an uncorrected significance of $p<0.001$.

Table 7. Brain regions showing significantly more activation during encoding with the easy task than during encoding with the hard task

\begin{tabular}{lllc}
\hline Brain region & Hemisphere & $\begin{array}{l}\text { Talairach coordinates } \\
(x, y, z)\end{array}$ & Brodmann area \\
\hline Superior frontal gyrus & Left & $-4,14,51$ & 8 \\
Middle frontal gyrus & Left & $-40,54,-16$ & 10 \\
Inferior frontal gyrus & Left & $-47,9,18$ & 44 \\
Precuneus & & $0,-67,62$ & 7 \\
Superior temporal gyrus & Left & $-60,-34,9$ & 22 \\
Inferior temporal gyrus & Left & $59,-13,-23$ & 20 \\
\hline
\end{tabular}

Regions were defined as significant if they included at least five functional voxels at an uncorrected significance of $p<0.001$.

easy distractor task, we found no evidence of additional neural circuits that were recruited to coordinate performance of the encoding task with performance of the more difficult auditory task. Even with the threshold dropped to $p<0.05$, the same occipital, parietal, and frontal regions were recruited in both tasks (Tables 5 and 6). These results are in contrast to the view that increasing task coordination demands require bringing on-line additional neural resources (D'Esposito et al., 1995) and instead suggest that the demands required for a more difficult task coordination can be met by the same regions active during a less demanding task coordination condition (see also Adcock et al., 2000; Bunge et al., 2001).

We then conducted ROI analyses that further examined activation related to task difficulty (within regions active during en- 


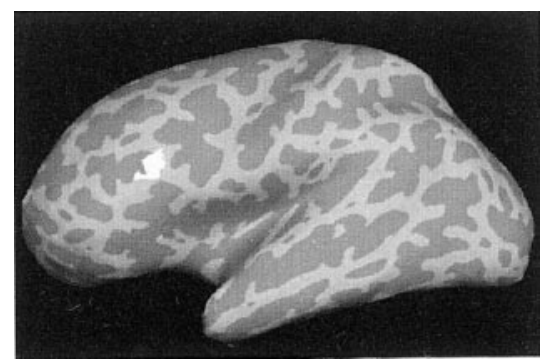

Figure 2. Effect of auditory task difficulty on activation at encoding. A region of left ventrolateral PFC (BA 44) showed a smaller peak percentage signal change during encoding with the hard task (2.1) than with the easy task $(2.5 ; p<0.001)$.

coding with both tasks). These analyses indicated that the magnitude of responses (maximum signal change) in the left inferior and left superior frontal regions were greater during encoding with the easy compared with the hard auditory task (Table 7, Fig. 2).

These results indicate that regions associated with encoding, including a region of the left ventrolateral PFC [Brodmann area (BA) 44; Talairach coordinate: $-48,19,25]$, showed less activation during concurrent performance of the hard task than during concurrent performance of the easy task. These data are in general agreement with the results of previous PET studies of divided attention (Shallice et al., 1994; Fletcher et al., 1995, 1998; Anderson et al., 2000; Iidaka et al., 2000), and suggest that some encoding processes were not being performed effectively during encoding with the hard auditory task.

To assure that this difference was attributable to differences in the encoding processes rather than to differences in the neural processes recruited for the auditory discrimination task, we conducted a control experiment in which nine participants performed only the auditory discrimination tasks. Voxel-based and ROI analyses indicated no modulation in the PFC or the MTL as a function of the difficulty of the secondary task. The only regions that showed significant modulation ( $p<0.01$, uncorrected) were the anterior cingulate gyrus (BA 31 ) and primary auditory cortex (BA 41, 42); these regions showed greater activation during performance of the hard task than the easy task.

The finding that the PFC reductions in activation were not related solely to the difficulty of the secondary task supports our hypothesis, and that proposed by previous researchers (Shallice et al., 1994; Fletcher et al., 1995; Iidaka et al., 2000), that the reductions in activation stem from differences in encoding processes performed with the two secondary tasks. What, then, are the neural processes that support successful encoding with the hard task and the easy task?

Functional MRI results: subsequent memory

To address this question, we conducted analyses of subsequent memory (as in Brewer et al., 1998; Fernandez et al., 1999; Wagner et al., 1999; Kirchhoff et al., 2000; Otten and Donchin, 2000; Davachi et al., 2001; Otten and Rugg, 2001). ROIs defined from the contrast of all encoding trials versus baseline indicated four regions that showed subsequent memory effects (i.e., greater signal change for subsequently remembered items than for subsequently forgotten items): two areas in the PFC [one in the left inferior PFC (BA 45/47) (Fig. 3A), and one in the right inferior PFC (BA 45) (Fig. 3B)] and two regions of the MTL [the anterior left hippocampus (Fig. $4 A$ ) and the left inferior middle parahippocampal gyrus (Fig. $4 B$ )]. In previous studies, activation in these regions, particularly the left PFC and MTL areas, also predicted subsequent memory (Wagner et al., 1999; Kirchhoff et al.,
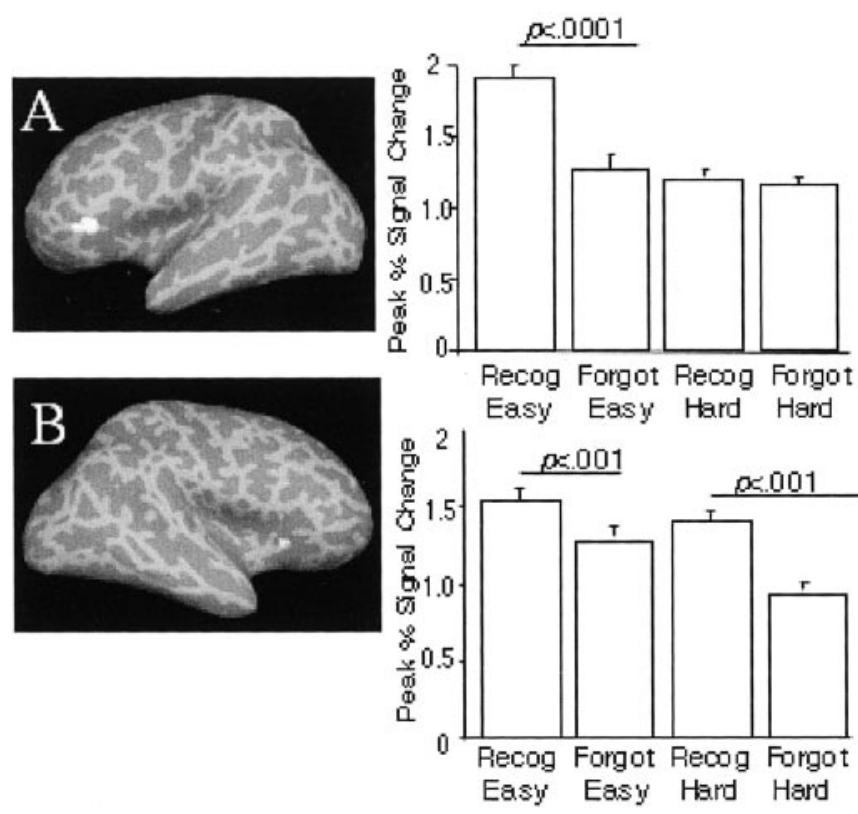

Figure 3. PFC activation at encoding related to retrieval success: Two regions showed activation related to subsequent memory. The left inferior PFC (BA 45/47; $A$ ) showed this effect only for items encoded with the easy task; the right inferior PFC (BA 45; $B)$ showed the relationship for items encoded with either the easy or the hard task.
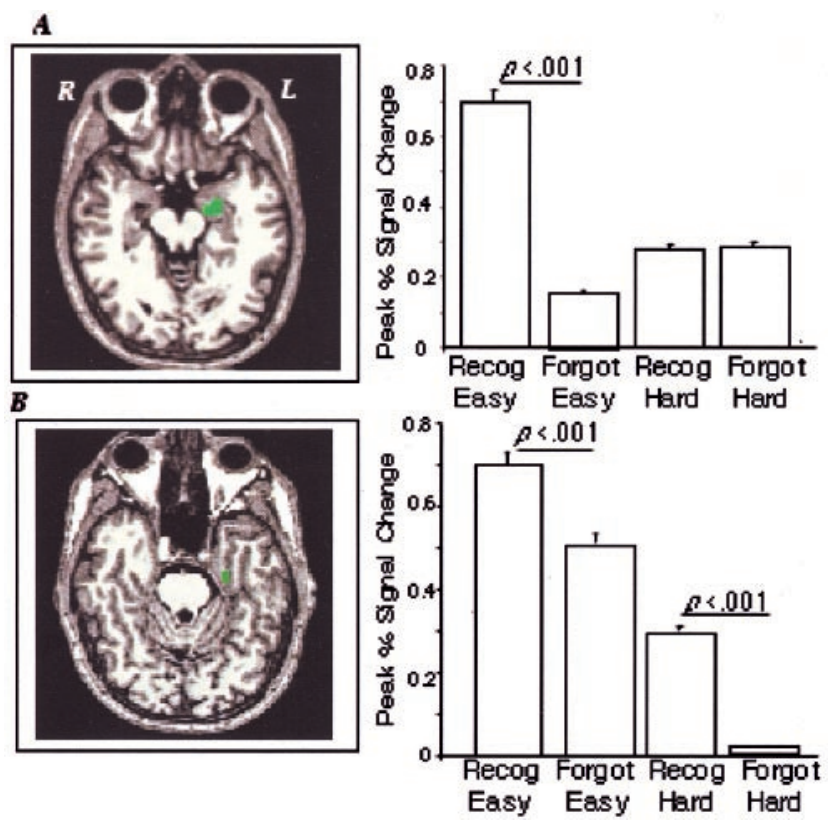

Figure 4. MTL activation at encoding relative to retrieval success. Two regions showed activation related to subsequent memory retrieval. The left anterior hippocampus $(A)$ showed the effect only for items encoded with the easy task, whereas the left parahippocampal gyrus $(B)$ showed the relationship for items encoded with either task. $R$, Right; $L$, left.

2000; Otten and Rugg, 2001; Davachi and Wagner, 2002; for review, see Paller and Wagner, 2002).

We then analyzed the activation in these ROIs to examine whether it was related to successful encoding (i.e., subsequent correct recognition) for items encoded with the easy and the hard auditory task, or only for items encoded in one of these conditions. The peak percentage of signal change (relative to baseline) in the left inferior PFC region (BA 45 and 47) predicted successful retrieval only for items encoded with the easy auditory task (Fig. 
$3 A$ ). Activation in this region did not differ for subsequently remembered versus forgotten items encoded with the hard auditory task. The findings were different for the area in the right inferior PFC (BA 45) (Fig. 3B). Here, activation predicted subsequent memory for items encoded with the easy task as well as for items encoded with the hard task.

Within the MTL, left anterior hippocampal activation (Fig. $4 A$ ) correlated with subsequent retrieval success only for items encoded with the easy task. In contrast, activation in the left inferior middle parahippocampal gyrus (Fig. $4 \mathrm{~B}$ ) predicted retrieval success for items encoded with either the easy or the hard task.

The results of the subsequent memory analyses indicated that the locus of signal change at encoding that predicted retrieval success for items encoded with the hard task comprised a subset of the areas in which activation changes predicted retrieval success for items encoded with the easy task. These data, together with the finding from the behavioral study that participants did not encode as much detailed information with the hard task, suggest that the formation of detailed, recollective memories depends on the left inferior PFC and left anterior hippocampus.

\section{fMRI results: effects of encoding condition on regions recruited during successful retrieval}

If, as the behavioral companion study and subsequent memory analyses suggested, participants formed qualitatively different types of memories for items encoded with the hard compared with the easy auditory task, it is possible that different brain regions were brought on-line during the retrieval of these traces.

\section{Successful retrieval: ROI analyses}

To address this issue, we defined ROIs based on the contrast of all retrieval (easy and hard) versus baseline. We then examined which of those regions showed greater activation during correct retrieval compared with incorrect retrieval. Four brain regions met this criterion. One region in the right inferior PFC (BA 44) (Fig. $5 A$ ) showed greater activation during retrieval of words encoded with the hard task compared with retrieval of words encoded with the easy task. This finding, together with the behavioral companion study data showing that memory traces lacking in detail occurred more frequently for items encoded with the hard versus the easy task, implicates the right inferior PFC as a component of the substrate for the sense of knowing or familiarity.

Two regions in the left PFC (BA 45 and BA 9 and 44) (Fig. $5 B, C)$ showed the opposite pattern: greater activation during retrieval of words encoded with the easy task. This result, together with the behavioral result that participants retrieved detailed memories more frequently for items encoded with the easy versus the hard task, identifies left PFC regions as part of the circuit underlying retrieval of detailed, recollective memories.

We also found one region in the left anterior hippocampus that showed greater activation during the retrieval of words encoded with the easy versus the hard task (Fig. 6). This finding is consistent with recent literature (Aggleton and Brown, 1999; Eldridge et al., 2000; Brown and Aggleton, 2001; Davachi et al., 2001; Strange et al., 2002) indicating that the hippocampus specifically supports in the retrieval of recollective, but not familiarity-based, memories. Despite recent claims that the parahippocampal gyrus is activated above baseline during retrieval of familiarity-based memories (Davachi et al., 2001; Yonelinas et al., 2001; Strange et al., 2002), we found no parahippocampal activation during retrieval.

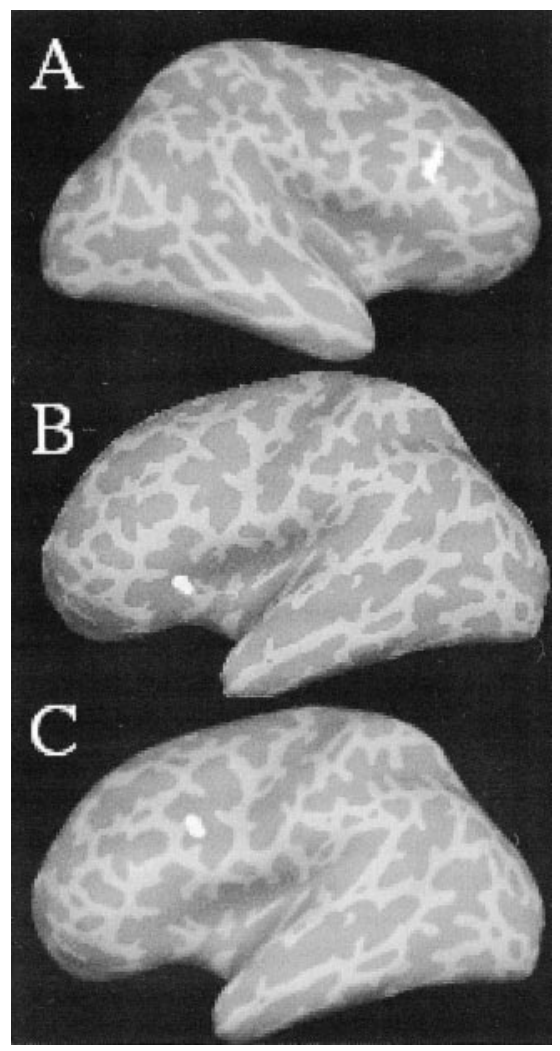

Figure 5. Prefrontal activation during successful retrieval related to encoding difficulty: A right inferior PFC region (BA 44; Talairach coordinate: $43,17,24 ; A$ ) showed greater activation during correct retrieval of words encoded with the hard task (1.5) compared with the easy task (1.2). In contrast, two regions of the left PFC (BA 45; Talairach coordinate: $-38,28,2 ; B$ ) and BA 9/44; Talairach coordinate: $-41,13,25 ; C)$ showed a greater percentage of signal change during retrieval of items encoded with the easy versus the hard task ( 1.0 and 0.75 , respectively, for region $B$, and 1.6 and 1.3 , respectively, for region $C ; p<0.001$ ).

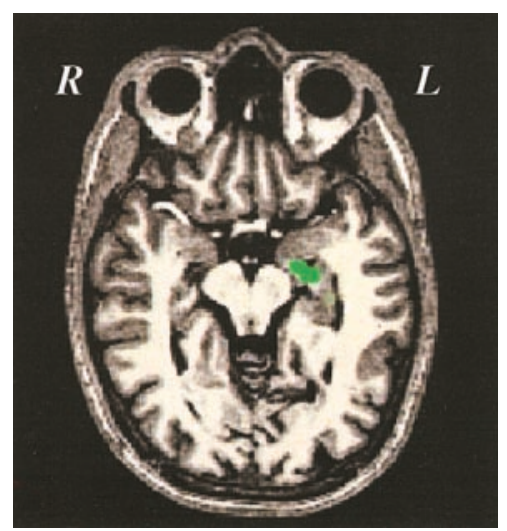

Figure 6. MTL activation during successful retrieval related to encoding difficulty. The left anterior hippocampus showed modulation similar to that seen for the left prefrontal regions: a greater percentage of signal change during retrieval of items encoded with the easy (0.57) than with the hard (0.17) task $(p<0.001)$. R, Right; $L$, left.

\section{Discussion}

The motivation for this study was the finding that although neural activation during encoding was reduced by divided attention (Shallice et al., 1994; Fletcher et al., 1995; Iidaka et al., 2000), individuals can, nevertheless, learn information when their attention is divided. The event-related design of this study allowed us to assess which brain regions support successful memory for- 
mation (i.e., successful encoding) when participants performed a hard or an easy secondary task. We also investigated whether the regions recruited for successful retrieval of information differed depending on whether that information had been learned with the hard task (favoring familiarity) or with the easy task (favoring recollection) at encoding.

Before addressing these two questions, we asked whether our divided-attention manipulation, like those used in previous PET studies (Shallice et al., 1994; Fletcher et al., 1995; Anderson et al., 2000; Iidaka et al., 2000), resulted in reductions in activation in regions implicated in episodic encoding. Consistent with previous PET studies, we found that encoding with the hard versus the easy distractor task reduced the activation in various $\mathrm{PFC}$ regions, including a region in the left ventrolateral PFC believed to play a role in maintenance of verbal information (Poldrack et al., 1999).

Like previous researchers (Shallice et al., 1994; Fletcher et al., 1995; Iidaka et al., 2000), we interpreted this reduction in activation as reflecting a degradation of encoding processes when participants performed the hard concurrent task. This view is consistent with the behavioral companion study reported here, and with extensive behavioral evidence that divided attention at encoding reduces the probability of successful retrieval (Craik et al., 1996; Naveh-Benjamin et al., 2000a,b), and in particular, reduces the ability to form rich, recollective memories (Yonelinas, 2001). It is also possible that encoding items with a hard secondary task reduces the explicit memory traces formed, forcing increased reliance on neural substrates supporting implicit memory formation (Shallice et al., 1994; Fletcher et al., 1995).

The convergence of our results using a constrained encoding task (i.e., "Decide whether each word is abstract or concrete") with those of previous PET studies using unconstrained encoding tasks (e.g., "Learn these word pairs") and cued recall (e.g., providing the first word of a word pair), suggests that the functional neuroimaging results generalize to a range of intentional encoding tasks. It will be worth examining in future studies whether this effect also generalizes to incidental encoding tasks (i.e., when participants are not aware that a memory task will follow).

\section{What brain regions support successful memory formation?}

We then investigated what brain regions supported successful encoding (i.e., encoding of items that participants later recognized correctly) in the hard task and the easy task. Previous research on encoding with full attention showed a relationship between successful encoding and magnitude of activation in the left PFC (Johnson et al., 1997; Wagner et al., 1999), hippocampus, and parahippocampal gyrus (Brewer et al., 1998; Fernandez et al., 1999; Wagner et al., 1999; Kirchhoff et al., 2000). Our results converge with these findings: The bilateral inferior PFC (left BA $45 / 47$, right BA 45), the left anterior hippocampus, and the left inferior middle parahippocampal gyrus all showed subsequent memory effects for items encoded with the easy task. Only a subset of these regions (right inferior PFC and left parahippocampal gyrus), however, related to subsequent memory for items encoded with the hard task. The regions that no longer supported successful encoding are regions that appear to be tied to the formation of detailed, recollective memories (Davachi et al., 2001; Strange et al., 2002), whereas the regions that continued to support successful encoding even with the concurrent hard secondary task are those that have been linked to formation of less detailed memories that evoke a "sense of knowing" or familiarity (Davachi et al., 2001; Strange et al., 2002). Our fMRI results, therefore, are consistent with the behavioral evidence presented here: Participants are more likely to form detailed memories for items encoded with the easy task than with the hard task.

\section{What brain regions support successful retrieval?}

We hypothesized that brain activation at retrieval could differ when retrieving qualitatively different memory traces (i.e., for detailed memories encoded with the easy task vs less detailed memories encoded with the hard task). Consistent with this hypothesis, we found a dissociation between the regions used to retrieve items encoded with the easy and with the hard task. In particular, we found greater activation in left PFC regions (BA 45; BA 9/44) for correctly retrieved items that had been encoded with the easy compared with the hard task, and greater activation in a right PFC (BA 44) region for items that had been encoded with the hard compared with the easy task. These laterality effects are consistent with recent neuroimaging studies suggesting that right-sided regions may be recruited when individuals are asked to make recognition judgments based on familiarity (Henson et al., 1999a,b, 2000; Dobbins et al., 2003), as may occur when memory traces are not detailed and include only familiarity information. In contrast, left-sided regions appear more important for retrieval of memories with rich detail (Johnson et al., 1997; Nolde et al., 1998a,b; Rugg et al., 1999; Ranganath et al., 2000).

A question has remained about whether these laterality effects represent differences in retrieval orientation (i.e., whether participants are attempting to retrieve or to monitor for detailed information or familiarity information) or differences in retrieval success (i.e., whether the products of the retrieval effort include recollective information or familiarity information). Our data support the interpretation that the laterality effects are based on differences in retrieval success. The retrieval scans intermixed items that had been encoded with the easy and hard tasks; thus, participants did not know the condition under which specific items had been encoded. Therefore, it is difficult to imagine that participants adopted different retrieval strategies for words encoded with the different tasks. It seems more plausible that the observed laterality effects were based on the qualitative nature of the memory trace that was retrieved (i.e., the amount of detail present). In other words, these results suggest that when different types of memory traces are formed at encoding [e.g., recollective vs familiarity-based or explicit vs implicit, as proposed by Fletcher et al. (1995) and Shallice et al. (1994)], different brain regions may be brought on-line later to allow the successful retrieval of those items.

It is unclear why these laterality effects occur: It is possible that right $\mathrm{PFC}$ regions are used during postretrieval monitoring, and thus are more critical for items with weaker traces, where they are closer to the boundary between what a person will accept as old versus assign as new (Henson et al., 2001; Yonelinas, 2002). Right prefrontal specialization is also found in studies of novelty (Menon et al., 2000), and a familiarity-based signal may be similar to that required for determining whether an object is novel. The left-sided activation found in the present study could result from semantic processing of the words; however, even studies with nonverbal stimuli have found left-sided prefrontal activation during retrieval of detailed information (Ranganath et al., 2000).

\section{Conclusion}

Using a divided-attention paradigm, we found that participants' memories of words encoded with a easy secondary task were more detailed (recollective) than those encoded with a hard task. A greater proportion of the words encoded with the hard task than the easy task relied on a less detailed sense of knowing or 
familiarity. fMRI indicated that encoding with the hard task showed less activation in PFC regions associated with episodic memory formation than encoding with the easy task. Only a subset of the brain regions that supported successful encoding with an easy secondary task continued to do so when encoding occurred with a hard secondary task. Activation in the left inferior PFC and the left anterior hippocampus predicted retrieval success only for items encoded with the easy task, whereas activation in the right inferior PFC and left inferior middle parahippocampal region predicted retrieval success for all items. Divided attention at encoding also affected the neural processes recruited for successful retrieval: We found a dissociation between brain regions preferentially used to retrieve items encoded with the easy task (left PFC and left anterior hippocampus) and those encoded with the hard task (right PFC). These results suggest that the brain regions that support successful encoding and successful retrieval vary depending on the detail of the memory trace. Familiarity-based traces lacking in detail may rely on right-sided PFC, whereas recollective traces that are rich in detail may rely more on left-sided PFC and the hippocampus.

\section{References}

Adcock R, Constable R, Gore J, Goldman-Rakic P (2000) Functional neuroanatomy of executive processes involved in dual-task performance. Proc Natl Acad Sci USA 97:3567-3572.

Aggleton JP, Brown MW (1999) Episodic memory, amnesia, and the hippocampal-anterior thalamic axis. Behav Brain Sci 22:425-489.

Anderson ND, Iidaka T, Cabeza R, Kapur S, McIntosh AR, Craik FI (2000) The effects of divided attention on encoding- and retrieval-related brain activity: a PET study of younger and older adults. J Cogn Neurosci 12:775-792.

Baddeley A (1984) Attention and retrieval from long-term memory. J Exp Psychol Gen 113:518-540.

Boynton GM, Engel SA, Glover GH, Heeger DJ (1996) Linear systems analysis of functional magnetic resonance imaging in human V1. J Neurosci 16:4207-4221.

Brewer JB, Zhao Z, Desmond JE, Glover GH, Gabrieli JD (1998) Making memories: brain activity that predicts how well visual experience will be remembered. Science 281:1185-1187.

Brown MW, Aggleton JP (2001) Recognition memory: what are the roles of the perirhinal cortex and hippocampus? Nat Rev Neurosci 2:51-61.

Bunge SA, Ochsner KN, Desmond JE, Glover GH, Gabrieli JD (2001) Prefrontal regions involved in keeping information in and out of mind. Brain 124:2074-2086.

Burock MA, Buckner RL, Woldorff MG, Rosen BR, Dale AM (1998) Randomized event-related experimental designs allow for extremely rapid presentation rates using functional MRI. NeuroReport 9:3735-3739.

Cox RW, Jesmanowicz A (1999) Real-time 3D image registration for functional MRI. Magn Reson Med 42:1014-1018.

Craik FI, Govoni R, Naveh-Benjamin M, Anderson ND (1996) The effects of divided attention on encoding and retrieval processes in human memory. J Exp Psychol Gen 125:159-180.

Craik FI, Naveh-Benjamin M, Ishaik G, Anderson ND (2000) Divided attention during encoding and retrieval: differential control effects? J Exp Psychol Learn Mem Cogn 26:1744-1749.

Dale AM (1999) Optimal experimental design for event-related fMRI. Hum Brain Mapp 8:109-114.

Dale AM, Buckner RL (1997) Selective averaging of rapidly presented individual trials using fMRI. Hum Brain Mapp 5:329-340.

Dale A, Greve D, Burock M (1999) Optimal stimulus sequences for eventrelated fMRI. Hum Brain Mapp 5:329-340.

Davachi L, Wagner AD (2002) Hippocampal contributions to episodic encoding: insights from relational and item-based learning. J Neurophysiol 88:982-990.

Davachi L, Maril A, Wagner AD (2001) When keeping in mind supports later bringing to mind: neural markers of phonological rehearsal predict subsequent remembering. J Cogn Neurosci 13:1059-1070.

Davachi L, Mitchell JP, Wagner AD (2003) Multiple routes to memory: distinct medial temporal lobe processes build item and source memories. Proc Natl Acad Sci USA 100:2157-2162.
D’Esposito M, Detre JA, Alsop DC, Shin RK, Atlas S, Grossman M (1995) The neural basis of the central executive system of working memory. Nature 378:279-281.

Dobbins IG, Rice HJ, Wagner AD, Schacter DL (2003) Retrieval orientation and success: separable neurocognitive components underlying episodic recognition. Neuropsychologia 41:318-333.

Eldridge LL, Knowlton BJ, Furmanski CS, Bookheimer SY, Engel SA (2000) Remembering episodes: a selective role for the hippocampus during retrieval. Nat Neurosci 3:1149-1152.

Fernandez G, Tendolkar I (2001) Integrated brain activity in medial temporal and prefrontal areas predicts subsequent memory performance: human declarative memory formation at the system level. Brain Res Bull 55:1-9.

Fernandez G, Effern A, Grunwald T, Pezer N, Lehnertz K, Dumpelmann M, Van Roost D, Elger CE (1999) Real-time tracking of memory formation in the human rhinal cortex and hippocampus. Science 285:1582-1585.

Fischl B, Sereno MI, Dale AM (1999) Cortical surface-based analysis. II. Inflation, flattening, and a surface-based coordinate system. NeuroImage 9:195-207.

Fischl B, Liu A, Dale AM (2001) Automated manifold surgery: constructing geometrically accurate and topologically correct models of the human cerebral cortex. IEEE Trans Med Imaging 20:70-80.

Fletcher PC, Frith CD, Grasby PM, Shallice T, Frackowiak RS, Dolan RJ (1995) Brain systems for encoding and retrieval of auditory-verbal memory. An in vivo study in humans. Brain 118:401-416.

Fletcher PC, Shallice T, Dolan RJ (1998) The functional roles of prefrontal cortex in episodic memory. I. Encoding. Brain 121:1239-1248.

Friston KJ, Zarahn E, Josephs O, Henson RN, Dale AM (1999) Stochastic designs in event-related fMRI. NeuroImage 10:607-619.

Gardiner JM, Java R (1993) Recognizing and remembering. In: Theories of memory (Collins AF, Gathercole SE, Conway MA, Morris PE, eds), pp 163-188. Hove, UK: Erlbaum.

Henson R, Rugg M, Shallice T, Josephs O, Dolan R (1999a) Recollection and familiarity in recognition memory: an event-related functional magnetic resonance imaging study. J Neurosci 19:3962-3972.

Henson R, Shallice T, Dolan RJ (1999b) Right prefrontal cortex and episodic memory retrieval: a functional MRI test of the monitoring hypothesis. Brain 122:1367-1381.

Henson R, Rugg MD, Shallice T, Dolan RJ (2000) Confidence in recognition memory for words: dissociating right prefrontal roles in episodic retrieval. J Cogn Neurosci 12:913-923.

Henson R, Shallice T, Rugg M, Fletcher P, Dolan R (2001) Functional imaging dissociations within right prefrontal cortex during episodic memory retrieval. Brain Cogn 47:79-81.

Iidaka T, Anderson ND, Kapur S, Cabeza R, Craik FI (2000) The effect of divided attention on encoding and retrieval in episodic memory revealed by positron emission tomography. J Cogn Neurosci 12:267-280.

Jacoby L (1991) A process dissociation framework? Separating automatic from intentional uses of memory. J Mem Language 30:513-541.

Johnson MK, Kounios J, Nolde SF (1997) Electrophysiological brain activity and memory source monitoring. NeuroReport 8:1317-1320.

Kirchhoff BA, Wagner AD, Maril A, Stern CE (2000) Prefrontal-temporal circuitry for episodic encoding and subsequent memory. J Neurosci 20:6173-6180.

Kucera H, Francis W (1967) Computational analysis of present-day American English. Providence, RI: Brown UP.

Mandler G (1980) Recognizing: the judgment of prior occurrence. Psychol Rev 87:252-271.

Mazziotta JC, Toga AW, Evans A, Fox P, Lancaster JA (1995) Probabilistic atlas of the human brain: theory and rationale for its development. NeuroImage 2:89-101.

Menon V, White CD, Eliez S, Glover GH, Reiss AL (2000) Analysis of a distributed neural system involved in spatial information, novelty, and memory processing. Hum Brain Mapp 11:117-129.

Naveh-Benjamin M, Guez J (2000) Effects of divided attention on encoding and retrieval processes: assessment of attentional costs and a componential analysis. J Exp Psychol Learn Mem Cogn 26:1461-1482.

Naveh-Benjamin M, Craik FI, Guez J, Dori H (1998) Effects of divided attention on encoding and retrieval processes in human memory: further support for an asymmetry. J Exp Psychol Learn Mem Cogn 24:1091-1104.

Naveh-Benjamin M, Craik FI, Perretta JG, Tonev ST (2000a) The effects of 
divided attention on encoding and retrieval processes: the resiliency of retrieval processes. Q J Exp Psychol A 53:609-625.

Naveh-Benjamin M, Craik FI, Gavrilescu D, Anderson ND (2000b) Asymmetry between encoding and retrieval processes: evidence from divided attention and a calibration analysis. Mem Cognit 28:965-976.

Nolde S, Johnson M, Raye C (1998a) The role of prefrontal cortex during tests of episodic memory. Trends Cogn Sci 2:399-405.

Nolde S, Johnson MK, D’Esposito M (1998b) Left prefrontal activation during episodic remembering: an event-related fMRI study. NeuroReport 9:3509-3514

Otten LJ, Donchin E (2000) Relationship between P300 amplitude and subsequent recall for distinctive events: dependence on type of distinctiveness attribute. Psychophysiology 37:644-661.

Otten LJ, Rugg MD (2001) When more means less: neural activity related to unsuccessful memory encoding. Curr Biol 11:1528-1530.

Paller KA, Wagner AD (2002) Observing the transformation of experience into memory. Trends Cogn Sci 6:93-102.

Poldrack RA, Wagner AD, Prull MW, Desmond JE, Glover GH, Gabrieli JD (1999) Functional specialization for semantic and phonological processing in the left inferior prefrontal cortex. NeuroImage 10:15-35.

Ranganath C, Johnson MK, D’Esposito M (2000) . Left anterior prefrontal activation increases with demands to recall specific perceptual information. J Neurosci 20:RC108(1-5).

Rugg MD, Fletcher PC, Chua PM, Dolan RJ (1999) The role of the prefrontal cortex in recognition memory and memory for source: an fMRI study. NeuroImage 10:520-529.
Shallice T, Fletcher P, Frith CD, Grasby P, Frackowiak RS, Dolan RJ (1994) Brain regions associated with acquisition and retrieval of verbal episodic memory. Nature 368:633-635.

Strange BA, Otten LJ, Josephs O, Rugg MD, Dolan RJ (2002) Dissociable human perirhinal, hippocampal, and parahippocampal roles during verbal encoding. J Neurosci 22:523-528.

Tulving E (1985) Memory and consciousness. Can Psychol 26:1-12.

Wagner AD, Schacter DL, Rotte M, Koutstaal W, Maril A, Dale A, Rosen BR, Buckner RL (1998) Building memories: remembering and forgetting verbal experiences as predicted by brain activity. Science 281:1188-1191.

Wagner AD, Koutstaal W, Schacter DL (1999) When encoding yields remembering: insights from event-related neuroimaging. Philos Trans R Soc Lond B Biol Sci 354:1307-1324.

Yonelinas AP (2001) Consciousness, control, and confidence: the 3 Cs of recognition memory. J Exp Psychol Gen 130:361-379.

Yonelinas AP (2002) The nature of recollection and familiarity: a review of 30 years of research. J Mem Language 46:441-517.

Yonelinas AP, Kroll NEA, Dobbins IG, Lazzara M, Knight RT (1998) Recollection and familiarity deficits in amnesia: convergence of remember/ know, process dissociation, and receiver operating characteristic data. Neuropsychology 12:1-17.

Yonelinas AP, Hopfinger JB, Buonocore MH, Kroll NEA, Baynes K (2001) Hippocampal, parahippocampal, and occipital-temporal contributions to associative and item recognition memory: an fMRI study. NeuroReport 12:359-363. 